\title{
Large non-radial propagation of a coronal mass ejection on 2011 January 24
}

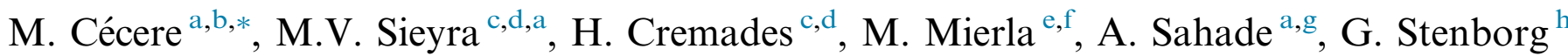 \\ A. Costa ${ }^{\text {a }}$, M.J. West ${ }^{\mathrm{e}}$, E. D'Huys ${ }^{\mathrm{e}}$ \\ ${ }^{a}$ Instituto de Astronomía, Teórica y Experimental, CONICET-UNC, Córdoba, Argentina \\ ${ }^{\mathrm{b}}$ Observatorio Astronómico de Córdoba, Universidad Nacional de Córdoba (UNC), Córdoba, Argentina \\ ${ }^{\mathrm{c}}$ Centro de Estudios para el Desarrollo Sustentable, Facultad Regional Mendoza-Universidad Tecnológica Nacional, Mendoza, Argentina \\ ${ }^{\mathrm{d}}$ Consejo Nacional de Investigaciones Científicas y Técnicas (CONICET), Argentina \\ ${ }^{\mathrm{e}}$ Solar-Terrestrial Center of Excellence - SIDC, Royal Observatory of Belgium, Brussels, Belgium \\ ${ }^{\mathrm{f}}$ Institute of Geodynamics of the Romanian Academy, Bucharest, Romania \\ ${ }^{\mathrm{g}}$ Facultad de Matemática, Astronomía, Física y Computación, UNC, Córdoba, Argentina \\ ${ }^{\mathrm{h}}$ Space Science Division, U.S. Naval Research Laboratory, Washington, DC 20375, USA
}

Received 26 May 2019; received in revised form 10 August 2019; accepted 28 August 2019

\begin{abstract}
Understanding the deflection of coronal mass ejections (CMEs) is of great interest to the space weather community because of their implications for improving the prediction of CME. This paper aims to shed light into the effects of the coronal magnetic field environment on CME trajectories. We analyze the influence of the magnetic environment on the early development of a particular CME event. On 2011 January 24 an eruptive filament was ejected in association with a CME that suffered a large deflection from its source region and expected trajectory. We characterize the $3 \mathrm{D}$ evolution of the prominence material using the tie-pointing/triangulation reconstruction technique on EUV and white-light images. To estimate the coordinates in 3D space of the apex of the CME we use a forwardmodeling technique that reproduces the large-scale structure of the flux rope-like CME, the Graduated Cylindrical Shell model. We found that the deflection amounts to $42^{\circ}$ in latitude and $20^{\circ}$ in longitude and that most of it occurs at altitudes below $4 R_{\odot}$. Moreover, we found a non-negligible deflection at higher altitudes. Combining images of different wavelengths with the extrapolated magnetic field obtained from a potential field source surface model we confirm the presence of two magnetic structures near the erupting event. The magnetic field environment suggests that field lines from the southern coronal hole act as a magnetic wall that produces the large latitudinal deflection; while a nearby pseudostreamer and a northward extension of the southern coronal hole may be responsible for the eastward deflection of the CME.
\end{abstract}

(C) 2019 COSPAR. Published by Elsevier Ltd. All rights reserved.

\section{Introduction}

Coronal mass ejections (CMEs) are the main drivers of geomagnetic storms. The study of their direction of

\footnotetext{
* Corresponding author.

E-mail address: mariana.cecere@unc.edu.ar (M. Cécere).
}

propagation throughout both the lower solar corona and interplanetary medium is a crucial first step to estimate their potential geo-effectiveness (see, e.g., Zhuang et al., 2017). The characterization of the coronal environment along the CME's early development is of utmost importance for finding potential structures that could influence the CMEs trajectory and hence make an assessment of 
the likelihood of an Earth encounter. CME events suffering deviations from the original direction of propagation (hereafter CME deflections) have been observed since the early days of space-borne coronagraphy (Hildner, 1977). Subsequently, several studies in both modeling and observations have focused on the investigation of CME deflections and their causes (e.g., Gui et al., 2011; Zuccarello et al., 2012; Liewer et al., 2015; Kay et al., 2016; Capannolo et al., 2017).

There are several solar magnetic structures which can affect the trajectory of a CME. Previous studies suggest that coronal holes $(\mathrm{CHs})$ act as magnetic walls that deflect CMEs away from them (e.g., Cremades et al., 2006; Gopalswamy et al., 2009; Panasenco et al., 2013) while the heliospheric current sheet (HCS), streamer-belt (SB) or pseudostreamers (PSs) may be favorable for channeling CMEs through these regions of low magnetic energy (Xie et al., 2009; Zuccarello et al., 2012; Lynch and Edmondson, 2013; Kay et al., 2013; Liewer et al., 2015; Yang et al., 2018). The magnetic pressure gradient over a CME would induce a net force in this direction (Gui et al., 2011; Shen et al., 2011; Liewer et al., 2015). Furthermore, the magnetic gradient perpendicular to the radial direction is larger at lower altitudes than at higher altitudes.

Nowadays, a myriad of space-borne instruments at several vantage points allows us to draw a consistent picture of the evolution of a CME from its very inception on the Sun and through its early development in the lower corona. Several studies have traced CME trajectories using observations from the imagers on board the twin spacecraft (S/C) of the Solar-Terrestrial Relations Observatory (STEREO; Kaiser et al., 2008) mission (e.g., Mierla et al., 2008; Panasenco et al., 2011); or by combining images from several missions: for instance using data from STEREO (ST), the Solar and Heliospheric Observatory (SOHO; Domingo et al., 1995), and the Solar Dynamics Observatory (SDO; Pesnell et al., 2012), as presented in e.g., Panasenco et al. (2013); and also including data provided by the SWAP (Sun Watcher with the APS and Image Processing; Halain et al., 2013; Seaton et al., 2013) imager on board the Project for OnBoard Autonomy 2 (PROBA2; Santandrea et al., 2013; see e.g., Mierla et al. (2013), Sarkar et al. (2019)).

In this paper we study a CME that occurred on 2011 January 24 that was deflected in both the latitudinal and the longitudinal direction with respect to the source region location. A filament erupting near a coronal hole was strongly deflected towards the equator and weakly deflected eastwards in direction towards a nearby PS while moving away from a northward extension of the southern polar $\mathrm{CH}$. To follow the trajectory of the CME components we use the images from EUV imagers (SDO/AIA and STEREO/EUVI) and the white-light images from coronagraphs (SOHO/LASCO-C2, STEREO/COR1 and STEREO/COR2). On the basis of these images we use the tie-pointing (TP) method to reconstruct the three-dimensional (3D) prominence coordinates and the graduated cylindrical shell (GCS) model to follow the apex of the CME. We analyze the deflection in terms of the coronal magnetic environment through which the prominence evolves, and how it influences the final direction of the CME.

\section{Event identification and methodology}

The analyzed event is a case study from an ongoing larger study on CME deflections, where we systematically analyze a number of cases to understand how the magnetic environment causes deviations in the trajectory of the CME from a radial direction. We analyze the period of time ranging between October 2010 and October 2011 when the solar activity was in the rising phase of solar cycle 24. One of the main advantages of this time period is that the STEREO spacecraft are almost in quadrature with respect to the Sun-Earth line (ST-A is $\sim 86^{\circ}$ away to the east and ST-B $\sim 92^{\circ}$ to the west), which facilitates simplified three-dimensional imaging of the Sun and low corona in combination with observatories located along the SunEarth line.

The investigation is carried out using extreme-ultraviolet (EUV) images from (i) the SWAP instrument on board PROBA2; (ii) the Extreme-Ultraviolet Imager (EUVI) of the SECCHI instrument suite (Sun-Earth Connection Coronal and Heliospheric Investigation; Howard et al., 2008) on board the twin STEREO S/C; and (iii) the AIA (Atmospheric Imaging Assembly; Lemen et al., 2012) on board SDO. In addition, we characterize the CME by means of white-light images from the SECCHI COR1 and COR2 coronagraphs on board both STEREO S/C and from LASCO-C2 (Large Angle Spectroscopic Coronagraph; Brueckner et al., 1995) on board SOHO.

In order to make a first selection of CMEs, we looked for events exhibiting large deflections -see definition below- with respect to the initial radial direction (as projected onto the plane of the sky of the instrument used). We first consider the eruptive filaments as reported by both the AIA Filament Eruption Catalog (McCauley et al., 2015) and the NASA CDAW prominence eruption catalog (Yashiro et al., 2013) and we determine their central position angles (PAs) prior to their eruptions observations in SDO/AIA $304 \AA$ images. The PA is the measure of projected angular distance determined in degrees counterclockwise from the solar north pole. To establish the associated CMEs we use information from the SOHO LASCO CME Catalog (Yashiro et al., 2004). We do not consider the CME PAs reported by this catalog, but rather we determine them in SOHO/LASCO-C2 images to avoid biases due to white-light shocks sometimes present in the images. Our baseline sample is then comprised of those events for which the absolute angular difference between CME PA and filament PA is greater than $20^{\circ}$. We chose a minimum of $20^{\circ}$ difference in agreement with the average 
unsigned deflection found by Cremades et al. (2006). We found 28 candidates with strong deflections out of a total of 143 prominence eruptions associated with CME events. It is worth noting that in some of these cases both the filament's and CME's PAs may be significantly different when a filament erupts in an asymmetric fashion (see e.g. Tripathi et al., 2006; Liu et al., 2009).

To characterize the "true" deflection, i.e., the deflection in 3D space, for each event we characterize the difference between the longitude and the latitude of the mid-point of the source region of the event (namely, the filament in its pre-eruptive stage as observed in $\mathrm{H} \alpha$ ) and those of the apex of the central axis of both the corresponding eruptive filament and CME (as observed in the EUV low corona and in the white-light corona).

In this work we analyze one case (January 24, 2011), that presents not only a large deviation between the source and the CME, but also a large deflection of the CME from its initial direction of propagation at significantly higher altitudes $\left(\sim(2-4) R_{\odot}\right)$ than is typically reported in coronal images between $(1-2.5) R_{\odot}$ (Zuccarello et al., 2012; Liewer et al., 2015; Kay et al., 2017). Gui et al. (2011) found deflection at exceptionally larger heights of $(2.5-4) R_{\odot}$.

To examine the deflection from the lower corona outward we use the images provided by the instruments on board the twin STEREO telescopes: EUVI (304 $)$ ), COR1, and COR2; in addition to observations from SDO/AIA (304 $\AA$ ) and SOHO/LASCO-C2.

To characterize the $3 \mathrm{D}$ evolution of the prominence material we use the tie-pointing reconstruction technique or triangulation, (see e.g., Inhester, 2006; Mierla et al., $2010)$ on the EUV images. The prominence material becomes the core of the CME as seen in the coronagraph images. The tie-pointing method uses a pair of images to trace the line-of-sight of a point selected in one image into the FOV of the second image. This line is called the epipolar line (see Inhester, 2006, for details on the epipolar geometry). The tie-pointing method is convenient when the triangulated structure is compact and well defined. Although the prominence is clearly visible in the FOV of SWAP, we were not able to apply the tie-pointing method to this data because the corresponding ST/EUVI $171 \AA$ images available to perform triangulation have very low cadence.

One of the most suitable and straightforward methods used to reproduce the large-scale structure of a flux ropelike $\mathrm{CME}$ is the forward-modeling technique. In this work, we use the Graduated Cylindrical Shell (GCS) model technique (Thernisien et al., 2006; Thernisien et al., 2009; Thernisien, 2011). This methodology allows us to estimate the coordinates of the apex of the CME in $3 \mathrm{D}$ space. Briefly, the model consists of a tubular section forming the main body of the structure attached to two cones that correspond to the "legs" of the CME. Only the outer envelope of the CME is modeled; i.e., there is no rendering of its internal structure. The parameters that can be derived by fitting the model to the CMEs observed in coronagraph data are: direction of propagation (longitude and latitude), height of the apex, the half angular width, the tilt angle around the axis of symmetry (with respect to the solar equator), and the aspect ratio of the model which sets the rate of expansion versus the height of the CME (selfsimilar expansion). Although several combinations of model parameters may seemingly fit the same CME, with proper constraints (e.g., using three viewpoints instead of two, considering the associated erupting prominence inclination, and a proper spacecraft-CME configuration) the GCS model is a valuable tool to ascertain the overall 3D properties of CMEs.

\section{Event description}

Fig. 1 shows a composite image of SDO/AIA $304 \AA$ and PROBA2/SWAP $174 \AA$ observations. Note that the field of view of the latter allows us to follow the feature of interest further out compared to SDO/AIA. The erupting prominence is seen above the southwest limb. The central location of the quiescent filament before eruption (henceforth source region) is indicated with a blue cross at the southwest hemisphere, at $\left(-53^{\circ}, 358^{\circ}\right)$ in the Carrington coordinate system at 10:11 UT on January 23, 2011. Its projected length is of the order of $100 \mathrm{Mm}$, as measured in $\mathrm{H} \alpha$ before the eruption. The filament starts to rise at $\sim 19: 00 \mathrm{UT}$, first releasing its western leg before finally erupting at 01:30 UT the next day. The ejected material can be seen to move towards equatorial latitudes from its source region, as can seen in the figure.

To the southwest of the filament there is a $\mathrm{CH}$, shown in Fig. 2, which exhibits an extension towards the solar

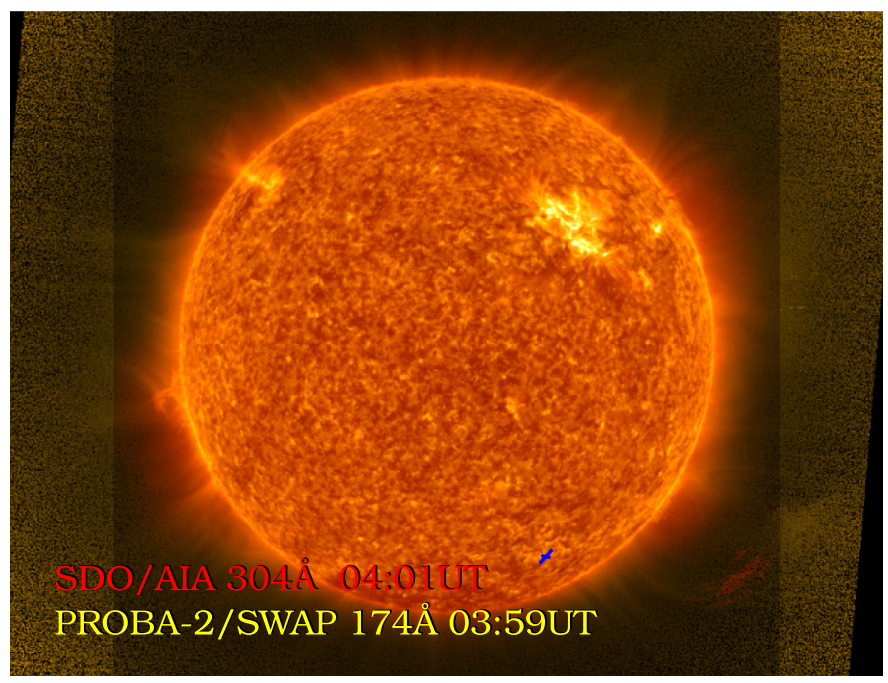

Fig. 1. Composite image using a SDO/AIA $304 \AA$ (reddish color) image and a PROBA2/SWAP $174 \AA$ (yellowish color) image that extends the view of the corona on 2011 January 24. The blue cross marks the central location of the source region. (For interpretation of the references to colour in this figure legend, the reader is referred to the web version of this article.) 


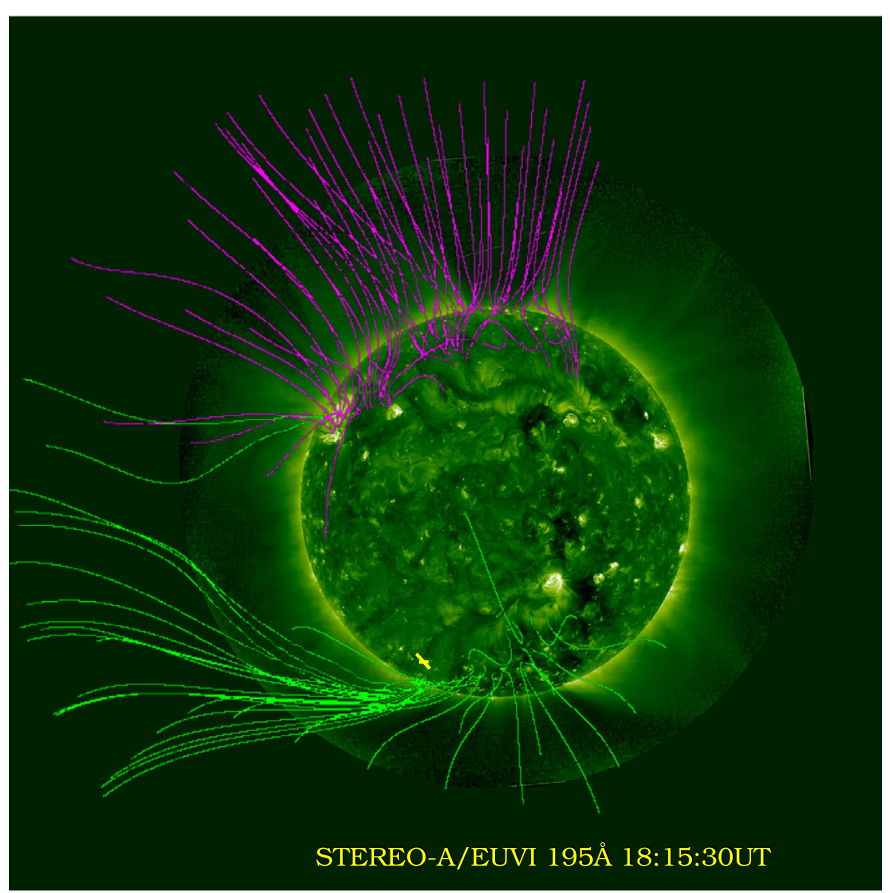

Fig. 2. Magnetic open field lines with positive polarity (green lines) overplotted on STEREO-A/EUVI 195 Aimage on 2011 January 23. The magenta lines represent magnetic field lines with negative polarity. The yellow cross marks the central location of the source region. (For interpretation of the references to colour in this figure legend, the reader is referred to the web version of this article.)

equator. The figure shows the $\mathrm{CH}$ magnetic field obtained by the Potential Field Source Surface (PFSS) model (Schrijver and De Rosa, 2003) superposed on a ST-A/ EUVI $195 \AA$ image obtained at 18:15UT on 2011 January 23 that has been processed with a multi-resolution, wavelet-based technique to enhance it (Stenborg et al., 2008). The green lines represent the open field lines emerging from the solar surface (the lines in magenta color represent open field lines with opposite polarity).

In order to trace the evolution of the prominence (see Fig. 3) we use the intrinsic function $\max$ from the
Interactive Data Language (IDL) package, in the 3D cube data (2D images along time), between 00:00 UT and 04:00 UT on January 24 from the Earth and STEREO-A views. The left panel shows a composite trace image with PROBA2/SWAP $174 \AA$ (greenish color) and SDO/AIA $304 \AA$ (reddish color) while the right panel shows the trace of the prominence in ST-A/EUVI 304 $\AA$ (reddish color). The green lines represent the coronal hole magnetic field.

Fig. 4 shows the magnetic field obtained by the PFSS model for the Earth view (top and middle panels) and for the ST-A view (bottom panel). Green and magenta lines represent open magnetic field lines and white ones are closed field lines. In the top panel of Fig. 4 we indicate with red letters the polarities (PP: positive polarity, NP: negative polarity) of the footpoints of the arcades (thick cyan lines between cyan arrows) that compose the base of the pseudostreamer located at $\left(-11^{\circ}, 294^{\circ}\right)$ and denoted as PS. In the middle and bottom panels of the same figure we point out with yellow numbers few thick green open lines that surround the PS.

These lines originate at the eastern edge of NOAA AR 11147 (lines 1-2) and the southern $\mathrm{CH}$ (lines 3-5).

Fig. 5 shows the prominence and the CME at different heights from the three points of view of the different telescopes. In the upper panels, from left to right, we see the prominence from the viewpoints of ST-B/EUVI, SDO/ AIA and ST-A/EUVI $(304 \AA)$ at $\sim 00: 27$ UT. The PA of the prominence measured from the AIA $304 \AA$ image at 00:27 UT was $218^{\circ}$ (see yellow angle in top middle panel). The middle panels show the CME in ST-B/COR1, SOHO/ LASCO-C2 and ST-A/COR1 respectively, at $\sim 03: 45 \mathrm{UT}$. We can see the core of the CME in the STEREO coronagraphs and the leading edge in the three images. In the bottom panels, we show the images of the CME at 06:50 UT in ST-B/COR2, SOHO/LASCO-C2 and ST-A/COR2. The difference between the PA of the prominence and the PA of the apex of the CME $\left(250^{\circ}\right.$ - see bottom middle panel of Fig. 5) is $32^{\circ}$. This measurement is however a first proxy

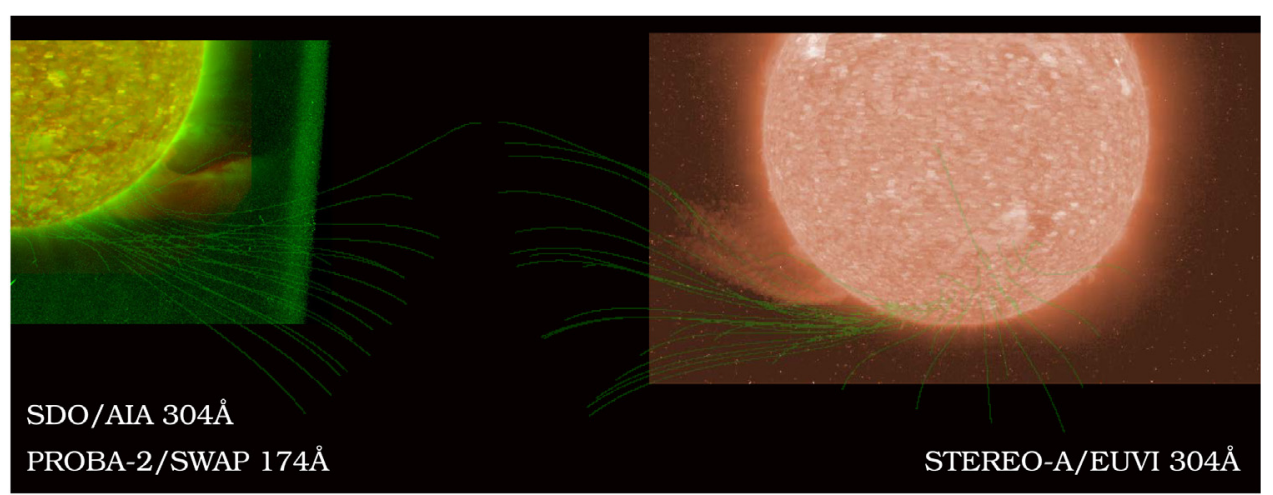

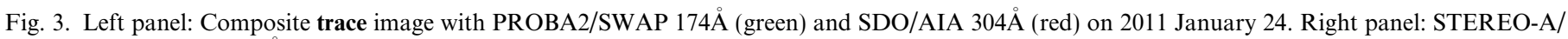
EUVI trace image at $304 \AA$ (red) on same day. On both panels, coronal hole magnetic field lines are overplotted as green lines. (For interpretation of the references to colour in this figure legend, the reader is referred to the web version of this article.) 


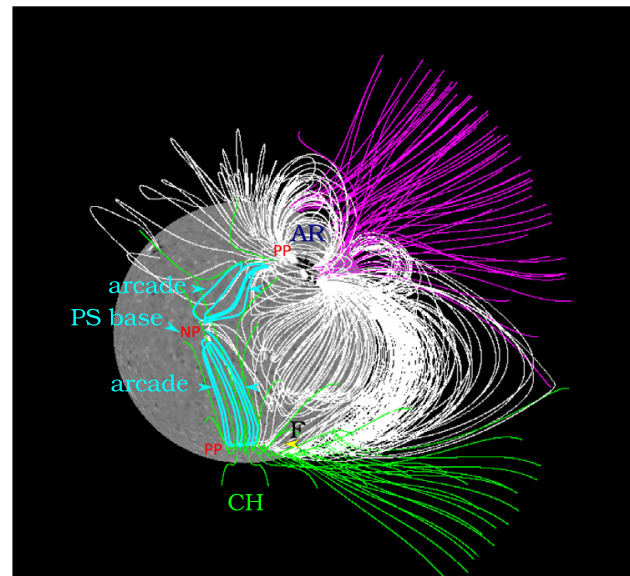

EARTH VIEW

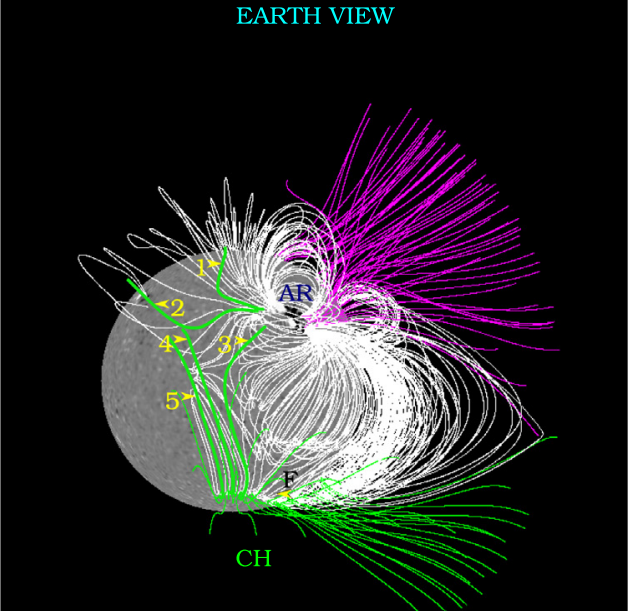

EARTH VIEW

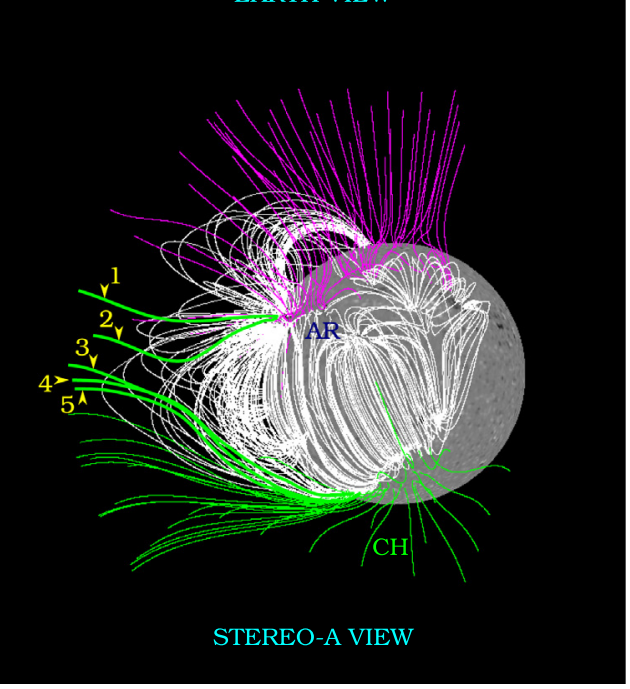

Fig. 4. PFSS extrapolation of the magnetic field on 2011 January 24. Top and middle panel: Earth view. Bottom panel: STEREO-A view. The coronal hole $(\mathrm{CH})$, pseudostreamer (PS), active region (AR) and the filament $(\mathrm{F})$ positions are indicated. The green lines are open magnetic field lines with positive polarity (PP) and the magenta ones are open magnetic field lines with negative polarity (NP). The yellow numbers indicate some of the thick green open lines that surround the PS. With cyan arrows we indicate some thick cyan closed lines that compose the arcades and the base (with negative polarity) of the PS. The white lines represent closed magnetic field lines. (For interpretation of the references to colour in this figure legend, the reader is referred to the web version of this article.)

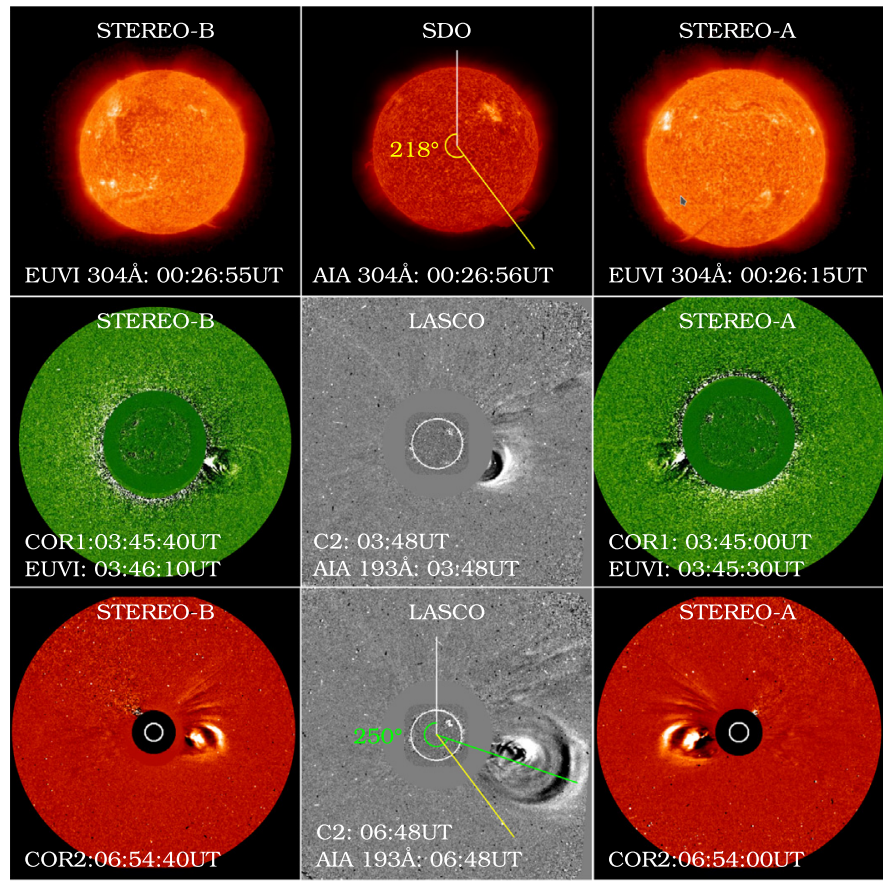

Fig. 5. Evolution of the prominence, CME core and leading edge on 2011 January 24. From left to right and top to bottom: ST-B/EUVI, SDO/AIA, ST-A/EUVI 304A at 00:26UT; ST-B/COR1, SOHO/LASCO-C2, ST-1/ COR1 at $\sim 03: 45 \mathrm{UT}$; ST-B/COR2, SOHO/LASCO-C2, ST-A/COR2 at $\sim 06: 50$ UT. Yellow lines indicate the PA of the prominence and the green one the PA of the apex of the leading edge. (For interpretation of the references to colour in this figure legend, the reader is referred to the web version of this article.)

of the deflection, given that it is determined from structures projected on the plane of the sky.

\section{Results}

\subsection{Latitudinal and longitudinal behavior}

As mentioned in Section 2, to obtain the real CME deflection from the position of the source region we track the $3 \mathrm{D}$ coordinates of the prominence, $\mathrm{CME}$ core and leading edge in time. Hence, for specific points in time we triangulate the part of the prominence which will become the core of the CME using pairs of EUV images of the low corona (SDO/AIA, ST-A/EUVI) and (SDO/AIA, ST-B/ EUVI). Then we follow its evolution at higher altitudes using images from the white-light coronagraphs $\mathrm{SOHO} /$ LASCO-C2, STEREO/COR1 and STEREO/COR2. In the upper panel of Fig. 6 we only show the portion of prominence triangulated with (SDO/AIA, ST-A/EUVI) $304 \AA$ at $\sim 00: 15$ UT. For each point in time (indicated by a white cross in the SDO/AIA image), to determine the uncertainty in the measurement of the extended structure, we select three different points along the epipolar line (indicated by colored crosses in ST-A/EUVI image). With these measurements we obtain averaged latitude, longitude and height values and calculate the error in the triangulation 


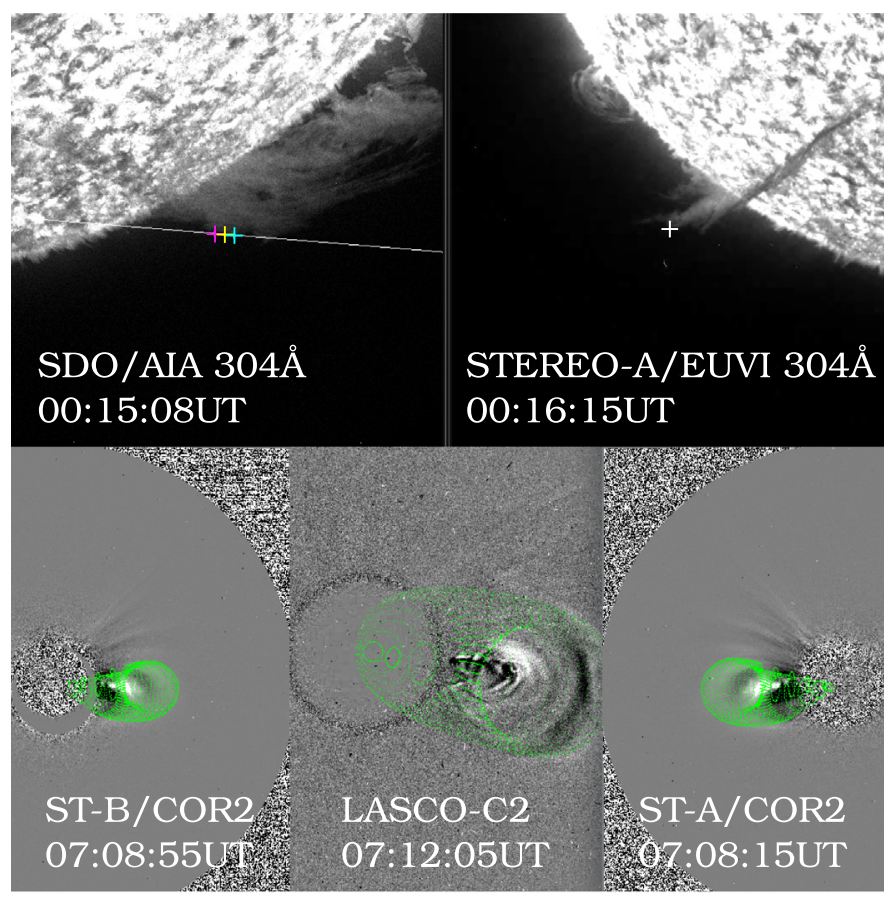

Fig. 6. Top panel: Triangulation of the prominence using images of SDO/ AIA $304 \AA$ and STEREO-A/EUVI $304 \AA$ at $\sim 00: 15$ UT on 2011, January 24 . On the right panel the white cross marks the structure selected on ST-A/EUVI image to triangulate. On the left panel, the colored crosses mark the 3 points selected along the epipolar white line to determine the uncertainty in the measurement to the extended structure. Bottom panel: GCS model of the CME using images of STEREO/COR2 and SOHO/ LASCO-C2 at $\sim 07: 10$ UT on 2011 January 24.

as the maximum value of the differences between the average value and each measurement. Also, we consider the contribution to the error in longitude $\operatorname{atan}\left(\right.$ depth $\left._{\text {error }} / R_{\mathrm{CME}}\right)$ due to the triangulation method, where depth $_{\text {error }}$ is the depth error $d s / \sin (\gamma)$ (see e.g., Mierla et al., 2009). The parameter $\gamma$ is the base angle between two spacecraft and $d s$ is the pointing error along the epipolar line in both images. To determine $d s$ we consider a pointing error along the epipolar line of 5 pixels in SDO/AIA, 2 pixels in STEREO/EUVI, 1 pixel in SOHO/LASCO-C2 and in STEREO/COR1 and 2 pixels in STEREO/COR2. These quantities are the result of the dispersion of pixels obtained when trying to triangulate the same point. After comparison of these two errors in longitude (due to the triangulation and to the depth error) we choose the larger one. These errors are shown as error bars of the filled circles in Fig. 7.

We use the GCS model to measure the CME apex coordinates of the leading edge using triplets of images from SOHO/LASCO-C2, ST-A/COR1 and -B; and SOHO/ LASCO-C2, ST-A/COR2 and -B. The bottom panel of Fig. 6 shows (in green contours) the fit obtained with STB/COR2, SOHO/LASCO-C2, and ST-A/COR2 images around $07: 10 \mathrm{UT}$. For each point in time we perform the best fit. To assess the error in the GCS determination of latitude, while keeping the other parameters fixed, we
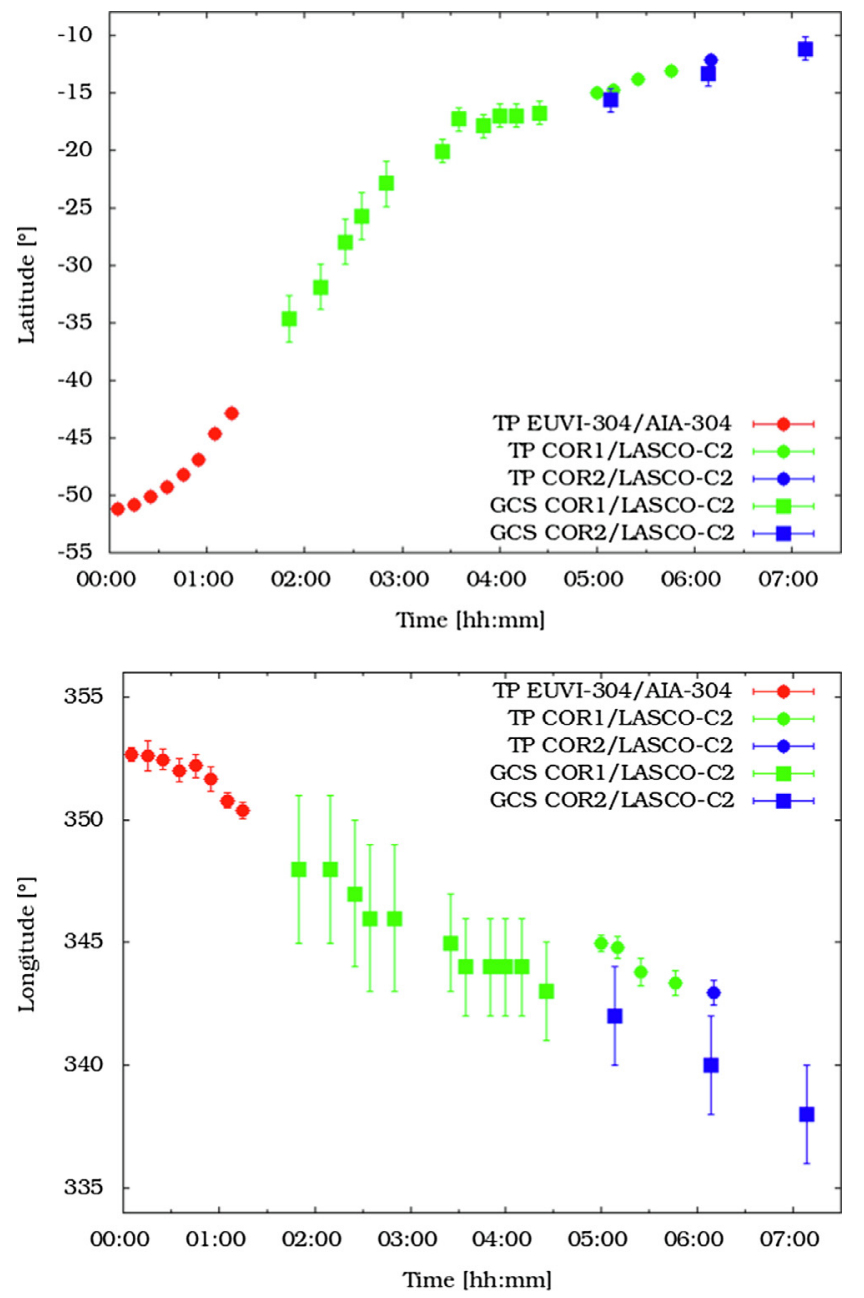

Fig. 7. Values of latitude and longitude vs. time on 2011 January 24. The circular symbols represent the averaged values obtained from the tiepointing method (on the prominence) and the squared symbols the values from the GCS model (corresponding to the CME apex).

iterated this parameter between lower and larger values until the fit was unacceptable. We repeated the same procedure for the longitude and height.

Fig. 7 shows the latitude and longitude averaged values obtained from applying the triangulation method to the prominence (red filled circles) using the pairs of images (SDO/AIA, ST-A/EUVI) and (SDO/AIA, ST-B/EUVI) as a function of time. Green circles represent the core averaged latitude and longitude values obtained from the triangulation method using (SOHO/LASCO-C2, ST-A/COR1) and (SOHO/LASCO-C2, ST-B/COR1) images, while blue circles correspond to the averaged values of latitude and longitude of the core from (SOHO/LASCO-C2, ST-A/ COR2) and (SOHO/LASCO-C2, ST-B/COR2) pairs. In addition, squares represent latitude and longitude of the apex of the CME leading edge obtained from the GCS model (green squares for ST-B/COR1, SOHO/LASCOC2, ST-A/COR1 and blue ones for ST-B/COR2, SOHO/ LASCO-C2, ST-A/COR2). From the figure we see that within a period of $6 \mathrm{~h}$ (after 00:00 UT) the prominence 
(circles) suffers a latitudinal deflection of $39^{\circ}$ (from $-51^{\circ}$ to $-12^{\circ}$ ) and a longitudinal deflection of $10^{\circ}$ (from $353^{\circ}$ to $343^{\circ}$ ). At 01:50 UT the leading edge (squares) becomes visible on the coronagraphs ST-A/COR1 and -B with central coordinates at $(-34,348)^{\circ}$. At 07:08 UT, the total latitudinal deflection of the CME apex throughout its journey in the white light corona amounts to $23^{\circ}$, and the longitudinal deflection to $10^{\circ}$. Then, the total deflection of the CME apex with respect to its source region is $42^{\circ}$ in latitude and $-20^{\circ}$ in longitude.

Fig. 8 shows the deflection in latitude and longitude as a function of height. We note that most of the latitudinal deflection occurs below 4 solar radii, while the longitude decreases smoothly with altitude.

\section{Discussion}

As described in the previous section, the deflection with respect to the source region of the prominence and its associated CME is in this case mainly in the latitudinal direction. From Fig. 8 we note that the growth rate in latitude changes at a height of $\sim 4 R_{\odot}$ and then becomes almost
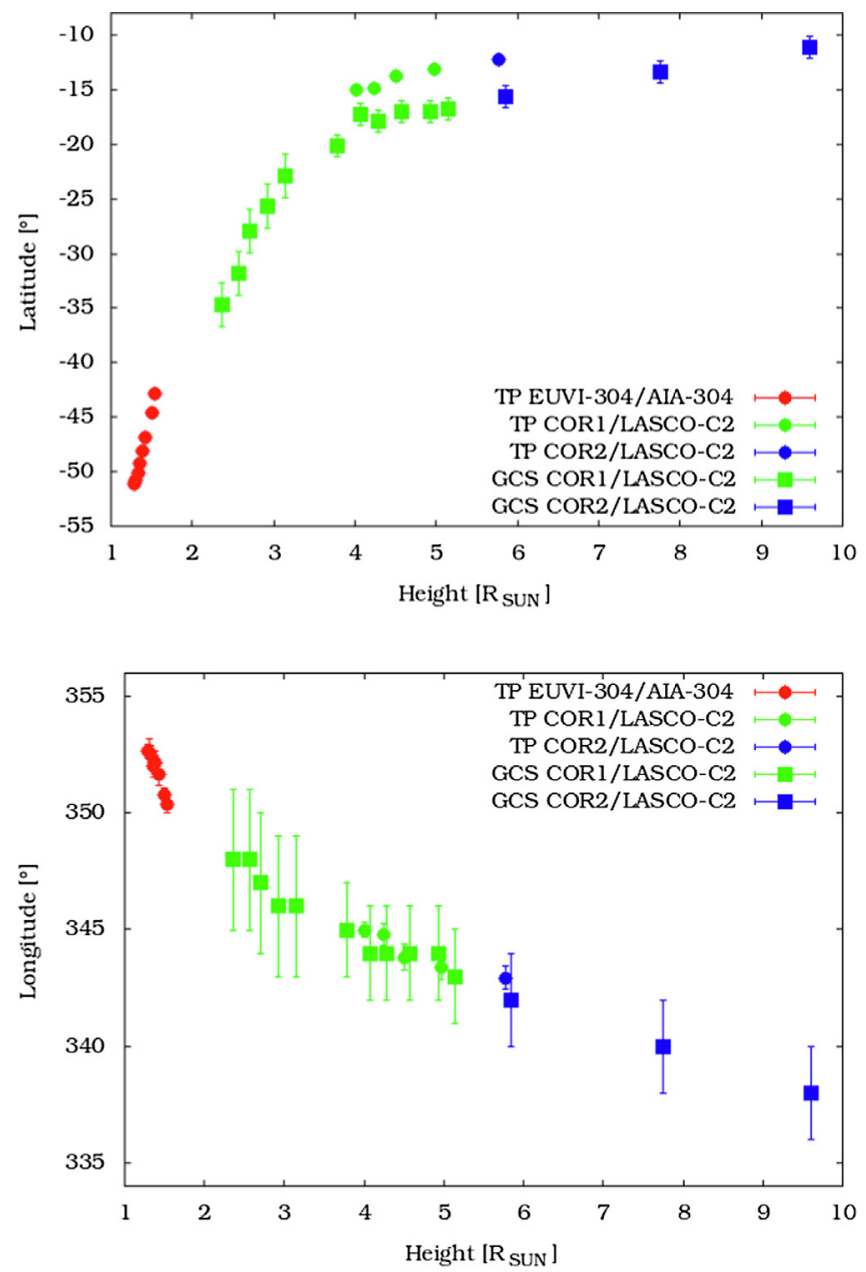

Fig. 8. Same as Fig. 7 but with latitude and longitude plotted as a function of height. constant. Until this altitude the CME suffers its greatest deflection, $17^{\circ}$ (from $-34^{\circ}$ to $-17^{\circ}$ ). As suggested by Shen et al. (2011), Gui et al. (2011), Zuccarello et al. (2012), Kay et al. (2013), Kay et al. (2017), Yang et al. (2018), the magnetic forces acting on the CME are relatively strong up to $\sim 4 R_{\odot}$. However, it has also been suggested (Mierla et al., 2008; Gui et al., 2011; Kay et al., 2015) that above this height the magnetic field could be still strong enough to deviate coronal eruptions. Indeed, as we see from Fig. 8 that above $4 R_{\odot}$ the apex of the leading edge suffers a deflection of only $6^{\circ}$, both in longitude and latitude.

In Fig. 9 we see the classic Carrington map of rotation 2106 obtained from Wilcox Solar Observatory Source Surface Synoptic Charts (Schatten et al., 1969; Altschuler and Newkirk, 1969; Hoeksema et al., 1983). The Carrington map shows the coronal magnetic field at $2.5 R_{\odot}$ calculated with a potential field model from photospheric field observations taken along a solar rotation. The field in the photosphere is assumed to be radial. The horizontal axis shows the Carrington longitude and the vertical axis is the latitude. The shaded areas are open magnetic field regions and the black solid line represents the heliospheric current sheet. The initial position of the source $\left(-53^{\circ}, 358^{\circ}\right)$ is denoted with a black circle, and the final position of the apex of the leading edge at $\left(-11^{\circ}, 338^{\circ}\right)$ with a red circle. Also, we show the location of an active region in close proximity (blue letters), the $\mathrm{CH}$ mentioned in Section 3 (light grey-shaded area), and the central position of the pseudostreamer at $\left(-11^{\circ}, 294^{\circ}\right)$ (cyan circle). The black arrow indicates the deflection in both latitude and longitude. We see that the CME trajectory deflects mostly latitudinally away from the southern part of the coronal hole (also see Figs. 1 and 3), and longitudinally towards the east moving away from the western part of the $\mathrm{CH}$

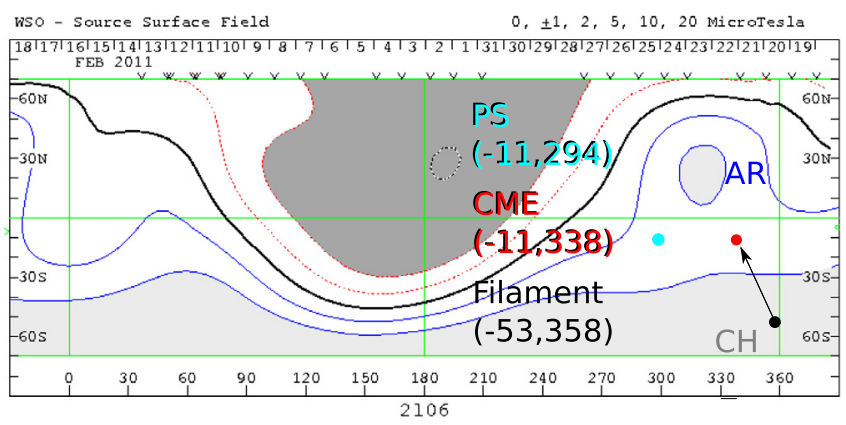

Fig. 9. Source Surface Synoptic Chart of Carrington rotation 2106 from Wilcox Solar Observatory. The black solid circle indicates the position of the source region and the red one the last central position of the CME leading edge. The black arrow indicates the CME deflection from its source region. The cyan circle represents the location on the solar surface where the magnetic fields from the eastern region of the AR and from the southern $\mathrm{CH}$ join and produce the double arcade of the PS. Colored text indicates the coordinates (latitude, Carrington longitude) of the three locations. (For interpretation of the references to colour in this figure legend, the reader is referred to the web version of this article.) 
(seen in Fig. 2) and and in the direction of the PS location (see Figs. 4 and 9).

In the first stages of the eruption the prominence erupts asymmetrically, as its northwestern leg is released before its southeastern leg. As pointed out by Liu (2008) and Liu et al. (2009) the strength and asymmetry of the background coronal magnetic field can constrain the trajectory of the filament eruption (see also review by Parenti, 2014). In our case, this asymmetric eruption would have a direction of propagation towards the southeast; however the influence of the coronal hole to the south seems to be stronger in this case, given that the final trajectory of the prominence is towards the northeast. Several authors have shown that open magnetic fields from coronal holes can act as magnetic walls causing the CMEs to perform non-radial motions away from these structures (e.g., Cremades et al., 2006; Gopalswamy et al., 2009; Panasenco et al., 2011; Mohamed et al., 2012; Panasenco et al., 2013), while a streamer or a pseudostreamer can resemble a potential well for CMEs (Xie et al., 2009; Panasenco and Velli, 2013; Lynch and Edmondson, 2013; Kay et al., 2013; Liewer et al., 2015; Yang et al., 2018). After the in-depth analysis of this event, we agree with the assumption that the (southern) $\mathrm{CH}$ seems to act as a magnetic wall, which is supported by Fig. 3. This would produce the strongest deflection of the CME towards the solar equator. At the same time, the CME is slightly deflected longitudinally towards the eastern PS, presumably because this region holds lower magnetic energy or because of the influence of the equatorward extension of the $\mathrm{CH}$ to the west of the source region, or a combination of both. According to Xie et al. (2009) the deflection of slow CMEs $(<400 \mathrm{~km} / \mathrm{s})$ has a preference towards potential wells. This CME is particularly slow, exhibiting a speed of the order of only $360 \mathrm{~km} / \mathrm{s}$.

\section{Conclusions}

With the goal of gaining a better understanding of CME deflections, we tracked the evolution of the threedimensional location of the CME on January 24, 2011; in particular that of its apex, core, and associated erupting prominence. The analyzed event showed a large deflection, not only with respect to its source region, but also to itself at high coronal altitudes as compared with lower coronal altitudes. Although most of the deflection takes place in the latitudinal direction, there is a notable deflection in longitude as well. We find that the main deflection occurs below the height of $4 R_{\odot}$, however, the CME still continues to clearly deflect in both latitudinal and longitudinal directions at larger distances from the Sun. By analyzing the magnetic field background through the PFSS model and the position of characteristic magnetic structures in close proximity of the filament such as a coronal hole and a pseudostreamer, we suggest that the main latitudinal deflection is due to the open magnetic field of the southern
$\mathrm{CH}$ and that the deflection to the east may be influenced by the presence of a low magnetic energy region located in the reconnection point of the pseudostreamer and to the western region of the $\mathrm{CH}$. To better understand how the magnetic environment affects the trajectory of the CME we are carrying out an extensive and systematic study comprising about 30 deflection events to be published in a forthcoming paper.

\section{Acknowledgements}

MC and VS acknowledge the PROBA2 Guest Investigator program grant received to carry out this work and the SWAP data provided by the PROBA2 team. MC, HC, and $\mathrm{AC}$ are members of the Carrera del Investigador Científico (CONICET). VS and AS are fellows of CONICET. AS thanks support from the Argentine Astronomy Association. MC and AS acknowledge support from ANPCyT under Grant No. PICT No. 2016-2480. MC, VS and AS also acknowledge support by SECYT-UNC Grant No. PC No 33620180101147CB. MM, MW and ED acknowledge support from the Belgian Federal Science Policy Office (BELSPO) through the ESA-PRODEX programme, Grant No. 4000120800. GS acknowledges the support from the NASA STEREO/SECCHI program NNG17PP27I and HC from UTN project UTI4915TC. The authors are thankful to the anonymous referees who helped us to improve the paper. The authors also acknowledge the use of the data from SDO/AIA, SOHO/LASCO-C2, STEREO/EUVI, STEREO/COR1, STEREO/COR2.

\section{References}

Altschuler, M.D., Newkirk, G., 1969. Magnetic fields and the structure of the solar corona. I: methods of calculating coronal fields. Solar Phys. 9, 131-149.

Brueckner, G.E., Howard, R.A., Koomen, M.J., Korendyke, C.M., Michels, D.J., Moses, J.D., Socker, D.G., Dere, K.P., Lamy, P.L., Llebaria, A., Bout, M.V., Schwenn, R., Simnett, G.M., Bedford, D.K., Eyles, C.J., 1995. The large angle spectroscopic coronagraph (LASCO). Solar Phys. 162, 357-402.

Capannolo, L., Opher, M., Kay, C., Landi, E., 2017. The deflection of the cartwheel CME: foreCAT results. Astrophys. J. 839, 37.

Cremades, H., Bothmer, V., Tripathi, D., 2006. Properties of structured coronal mass ejections in solar cycle 23. Adv. Space Res. 38, 461-465.

Domingo, V., Fleck, B., Poland, A.I., 1995. The SOHO mission: an overview. Sol. Phys. 162, 1-37.

Gopalswamy, N., Mäkelä, P., Xie, H., Akiyama, S., Yashiro, S., 2009. CME interactions with coronal holes and their interplanetary consequences. J. Geophys. Res. (Space Physics) 114, A00A22.

Gui, B., Shen, C., Wang, Y., Ye, P., Liu, J., Wang, S., Zhao, X., 2011. Quantitative analysis of CME deflections in the corona. Solar Phys. 271, 111-139.

Halain, J.-P., Berghmans, D., Seaton, D.B., Nicula, B., De Groof, A., Mierla, M., Mazzoli, A., Defise, J.-M., Rochus, P., 2013. The SWAP EUV imaging telescope. Part II: in-flight performance and calibration. Solar Phys. 286, 67-91.

Hildner, E., 1977. Mass ejections from the solar corona into interplanetary space. In: Shea, M.A., Smart, D.F., Wu, (Eds.), Study of Travelling Interplanetary Phenomena, Vol. 71 of Astrophysics and Space Science Library, pp. 3-20. 
Hoeksema, J.T., Wilcox, J.M., Scherrer, P.H., 1983. The structure of the heliospheric current sheet $-1978-1982$. J. Geophys. Res. 88, 9910 9918.

Howard, R.A., Moses, J.D., Vourlidas, A., Newmark, J.S., Socker, D.G., Plunkett, S.P., Korendyke, C.M., Cook, J.W., Hurley, A., Davila, J. M., et al., 2008. Sun earth connection coronal and heliospheric investigation (SECCHI). Space Sci. Rev. 136, 67-115.

Inhester, B., 2006. Stereoscopy basics for the STEREO mission. arXiv Astrophysics e-prints.

Kaiser, M.L., Kucera, T.A., Davila, J.M., St, O.C., Cyr, M. Guhathakurta, Christian, E., 2008. The STEREO mission: an introduction. Space Sci. Rev. 136, 5-16.

Kay, C., Opher, M., Colaninno, R.C., Vourlidas, A., 2016. Using foreCAT deflections and rotations to constrain the early evolution of CMEs. Astrophys. J. 827, 70.

Kay, C., Opher, M., Evans, R.M., 2013. Forecasting a coronal mass ejection's altered trajectory: foreCAT. Astrophys. J. 775, 5.

Kay, C., Opher, M., Evans, R.M., 2015. Global trends of CME deflections based on CME and solar parameters. Astrophys. J. 805, 168.

Kay, C., Gopalswamy, N., Xie, H., Yashiro, S., 2017. Deflection and rotation of CMEs from active region 11158. Solar Phys. 292, 78.

Lemen, J.R., Title, A.M., Akin, D.J., Boerner, P.F., Chou, C., Drake, J. F., Duncan, D.W., Edwards, C.G., Friedlaender, F.M., Heyman, G. F., et al., 2012. The atmospheric imaging assembly (AIA) on the solar dynamics observatory (SDO). Solar Phys. 275, 17-40.

Liewer, P., Panasenco, O., Vourlidas, A., Colaninno, R., 2015. Observations and analysis of the non-radial propagation of coronal mass ejections near the sun. Solar Phys. 290, 3343-3364.

Liu, Rui, Alexander, David, Gilbert, Holly R., 2009. Asymmetric eruptive filaments. Astrophys. J. 691 (2), 1079-1091.

Liu, Y., 2008. Magnetic field overlying solar eruption regions and kink and torus instabilities. Astrophys. J. Lett. 679, L151.

Liu, Y., Su, J., Xu, Z., Lin, H., Shibata, K., Kurokawa, H., 2009. New observation of failed filament eruptions: the influence of asymmetric coronal background fields on solar eruptions. Astrophys. J. Lett. 696, L70-L73.

Lynch, B.J., Edmondson, J.K., 2013. Sympathetic magnetic breakout coronal mass ejections from pseudostreamers. Astrophys. J. 764, 87.

McCauley, P.I., Su, Y.N., Schanche, N., Evans, K.E., Su, C., McKillop, S., Reeves, K.K., 2015. Prominence and filament eruptions observed by the solar dynamics observatory: statistical properties, kinematics, and online catalog. Solar Phys. 290, 1703-1740.

Mierla, M., Inhester, B., Antunes, A., Boursier, Y., Byrne, J.P., Colaninno, R., Davila, J., de Koning, C.A., Gallagher, P.T., Gissot, S., Howard, R.A., Howard, T.A., Kramar, M., Lamy, P., Liewer, P. C., Maloney, S., Marqué, C., McAteer, R.T.J., Moran, T., Rodriguez, L., Srivastava, N., St, O.C., Cyr, G., Stenborg, M., Temmer, A., Thernisien, A., Vourlidas, M.J., West, B.E. Wood, Zhukov, A.N., 2010. On the 3-D reconstruction of coronal mass ejections using coronagraph data. Ann. Geophys. 28, 203-215.

Mierla, M., Inhester, B., Marqué, C., Rodriguez, L., Gissot, S., Zhukov, A.N., Berghmans, D., Davila, J., 2009. On 3D Reconstruction of coronal mass ejections: I. Method description and application to SECCHI-COR data. Solar Phys. 259, 123-141.

Mierla, M., Seaton, D.B., Berghmans, D., Chifu, I., De Groof, A., Inhester, B., Rodriguez, L., Stenborg, G., Zhukov, A.N., 2013. Study of a prominence eruption using PROBA2/SWAP and STEREO/EUVI Data. Solar Phys. 286, 241-253.

Mierla, M., Davila, J., Thompson, W., Inhester, B., Srivastava, N., Kramar, M., St, O.C., Cyr, G. Stenborg, Howard, R.A., 2008. A quick method for estimating the propagation direction of coronal mass ejections using STEREO-COR1 images. Solar Phys. 252, 385-396.

Mohamed, A.A., Gopalswamy, N., Yashiro, S., Akiyama, S., Mäkelä, P., Xie, H., Jung, H., 2012. The relation between coronal holes and coronal mass ejections during the rise, maximum, and declining phases of Solar Cycle 23. J. Geophys. Res. (Space Physics) 117, A01103.
Panasenco, O., Velli, M., 2013. Coronal pseudostreamers: source of fast or slow solar wind?. Solar Wind 13 (1539), 50-53.

Panasenco, O., Martin, S.F., Velli, M., Vourlidas, A., 2013. Origins of rolling, twisting, and non-radial propagation of eruptive solar events. Solar Phys. 287, 391-413.

Panasenco, O., Martin, S., Joshi, A.D., Srivastava, N., 2011. Rolling motion in erupting prominences observed by STEREO. J. Atmos. Solar Terr. Phys. 73, 1129-1137.

Parenti, Susanna, 2014. Solar prominences: observations. Living Rev. Sol. Phys. 11 (1), 1.

Pesnell, W.D., Thompson, B.J., Chamberlin, P.C., 2012. The solar dynamics observatory (SDO). Sol. Phys. 275, 3-15.

Santandrea, S., Gantois, K., Strauch, K., Teston, F., Tilmans, E., Baijot, C., Gerrits, D., De Groof, A., Schwehm, G., Zender, J., 2013. PROBA2: mission and spacecraft overview. Solar Phys. 286, 5-19.

Sarkar, R., Srivastava, N., Mierla, M., West, M.J., D’Huys, E., 2019. Evolution of the coronal cavity from the quiescent to eruptive phase associated with coronal mass ejection. Astrophys. J. 875, 101.

Schatten, K.H., Wilcox, J.M., Ness, N.F., 1969. A model of interplanetary and coronal magnetic fields. Solar Phys. 6, 442-455.

Schrijver, C.J., De Rosa, M.L., 2003. Photospheric and heliospheric magnetic fields. Solar Phys. 212, 165-200.

Seaton, D.B., Berghmans, D., Nicula, B., Halain, J.-P., De Groof, A., Thibert, T., Bloomfield, D.S., Raftery, C.L., Gallagher, P.T., Auchère, F., Defise, J.-M., D’Huys, E., Lecat, J.-H., Mazy, E., Rochus, P., Rossi, L., Schühle, U., Slemzin, V., Yalim, M.S., Zender, J., 2013. The SWAP EUV imaging telescope. Part I: instrument overview and preflight testing. Solar Phys. 286, 43-65.

Shen, C., Wang, Y., Gui, B., Ye, P., Wang, S., 2011. Kinematic evolution of a slow CME in corona viewed by STEREO-B on 8 October 2007. Solar Phys. 269, 389-400.

Stenborg, G., Vourlidas, A., Howard, R.A., 2008. A fresh view of the extreme-ultraviolet corona from the application of a new imageprocessing technique. Astrophys. J. 674, 1201-1206.

Thernisien, A., 2011. Implementation of the graduated cylindrical shell model for the three-dimensional reconstruction of coronal mass ejections. Astrophys. J. Suppl. 194, 33.

Thernisien, A., Vourlidas, A., Howard, R.A., 2009. Forward modeling of coronal mass ejections using STEREO/SECCHI data. Solar Phys. 256, 111-130.

Thernisien, A.F.R., Howard, R.A., Vourlidas, A., 2006. Modeling of flux rope coronal mass ejections. Astrophys. J. 652, 763-773.

Tripathi, D., Isobe, H., Mason, H.E., 2006. On the propagation of brightening after filament/prominence eruptions, as seen by SoHOEIT. Astron. Astrophys. 453 (3), 1111-1116.

Xie, H., St, O.C., Cyr, N., Gopalswamy, S., Yashiro, J., Krall, M. Kramar, Davila, J., 2009. On the origin, 3D structure and dynamic evolution of CMEs near solar minimum. Solar Phys. 259, 143-161.

Yang, J., Dai, J., Chen, H., Li, H., Jiang, Y., 2018. Filament eruption with a deflection of nearly 90 degrees. Astrophys. J. 862, 86.

Yashiro, S., Gopalswamy, N., Michalek, G., St, O.C., Cyr, S.P., Plunkett, N.B. Rich, Howard, R.A., 2004. A catalog of white light coronal mass ejections observed by the SOHO spacecraft. J. Geophys. Res. (Space Physics) 109, A07105.

Yashiro, S., Gopalswamy, N., Makela, P., Akiyama, S., Sterling, A.C., 2013. An automatic detection technique for prominence eruptions and surges using SDO/AIA images. AAS/Solar Physics Division Meeting, 100.99 .

Zhuang, B., Wang, Y., Shen, C., Liu, S., Wang, J., Pan, Z., Li, H., Liu, R., 2017. The significance of the influence of the CME deflection in interplanetary space on the CME arrival at earth. Astrophys. J. 845, 117.

Zuccarello, F.P., Bemporad, A., Jacobs, C., Mierla, M., Poedts, S., Zuccarello, F., 2012. The role of streamers in the deflection of coronal mass ejections: comparison between STEREO three-dimensional reconstructions and numerical simulations. Astrophys. J. 744, 66. 